\title{
Fator von Willebrand e Disfunção Endotelial Pulmonar. Implicações Prognósticas
}

\author{
Antonio Augusto B. Lopes, Nair Yukie Maeda, Sérgio Paulo Bydlowski
}

São Paulo, SP

Objetivo - Avaliar alterações quantitativas e estruturais do fator von Willebrand ( $f v W$ ) circulante em 40 pacientes com hipertensão pulmonar pré-capilar e verificar possíveis implicações prognósticas dos resultados iniciais, em um ano de seguimento.

Métodos - A atividade antigênica plasmática do fator von Willebrand (vWF:Ag) foi analisada por imunoeletroforese. A concentração de multímeros de baixo peso molecular em relação ao total de multímeros do $f v W$ (MBPM\%) e o grau de fragmentação de sua subunidade principal foram analisados por Western immunoblotting.

Resultados - Em 40 pacientes, observamos aumento significativo de $v W F: A g$ em relação aos controles ( $p<0,001)$. Também foi observado aumento de MBPM\% ( $p<0,001)$, além de degradação patológica da subunidade do fvW $(p<0,05)$. Em 11 pacientes que evoluíram para óbito no $1^{\circ}$ ano, os valores iniciais de $v W F: A g$ foram 95\% superiores em relação aos sobreviventes ( $p<0,001)$. Os não sobreviventes tiveram ainda $31 \%$ de aumento em MBPM\% ( $p<0,005)$. A variável MBPM\% foi selecionada por regressão logística $(p<0,05)$, como preditor de óbito no primeiro ano de evolução.

Conclusão - As alterações do fvW circulante, consideradas como índices de lesão endotelial, têm valor prognóstico em hipertensão arterial pulmonar, com potencial impacto em decisões terapêuticas, como a indicação de transplante cardiopulmonar.

Palavras-chave: fator von Willebrand, hipertensão pulmonar, célula endotelial

\section{von Willebrand Factor and Pulmonary Vascular Endothelial Cell Dysfunction. Prognostic Implications}

Purpose - To analyze quantitative and structural changes in circulating von Willebrand factor ( $v W F)$ in 40 precapillary pulmonary hypertensive patients, as an attempt to identify possible correlations between endothelial cell dysfunction and patient short-term (one year) survival.

Methods - Plasma antigenic activity of vWF (vWF:Ag) was analyzed by immunoelectrophoresis. The relative concentration of $v W F$ low molecular weight multimers (LMWM\%) and the composition of $v W F$ subunitwere determined by densitometric analysis of Western blots.

Results - vWF:Ag was importantly increased in patients in comparison with normals $(p<0.001)$. Patients also had increased LMWM\% ( $p<0.001)$ and increased degradation of $v W F$ main subunit $(p<0.05)$. At the beginning of the study, nonsurvivors $(N=11)$ had higher $v W F: A g(p<0.001)$ and LMWM\% ( $p<0.005)$ values in comparison with survivors. LMWM\% was selected by logistic regression analysis as a predictor of death during the first year of follow-up ( $p<0.05)$.

Conclusion - Marked changes in circulating $v W F$ likely reflect extensive pulmonary vascular endothelial cell dysfunction and are associated with poor short-term prognosis in pulmonary hypertension.

Key-words: von Willebrand factor, pulmonary hypertension, endothelial cell

Arq Bras Cardiol, volume 70 (nº 3), 141-145, 1998

O fator von Willebrand (fvW), também designado por fator VIII endotelial, é uma glicoproteína de estrutura

Instituto do Coração, Hospital das Clínicas da FMUSP e Fundação Pró-Sangue Hemocentro de São Paulo

Correspondência: Antonio Augusto B. Lopes - Laboratório de Pesquisa - Fundação Pró-Sangue Hemocentro de São Paulo - Av. Dr. Enéas C. Aguiar, 155 - $1^{\circ}$ - PAMB - 05403-000 - São Paulo, SP

Recebido para publicação em 13/10/97

Aceito em 7/1/98 multimérica, sintetizada exclusivamente por células endoteliais e megacariócitos. Além de atuar na adesão e agregação plaquetária, como molécula de adesão, participa como transportador do fator VIII da coagulação (de origem hepática), aumentando consideravelmente sua meia-vida plasmática ${ }^{1}$. O fvW possui subunidades idênticas, com migração eletroforética de $225 \mathrm{kDa}$. Essas subunidades se interligam para formar multímeros de tamanhos variados, chegando a $20.000 \mathrm{kDa}^{2,3}$. Em condições fisiológicas, a proteína endotelial é secretada constitutivamente, tanto para a 
membrana basal como para o sangue. Uma vez em circulação, os grandes polímeros sofrem uma limitada proteólise "fisiológica", dando origem a moléculas menores, com massa entre 500 (dímeros) e $15.000 \mathrm{kDa}^{4}$. Ainda em condições fisiológicas, parte da proteína endotelial é armazenada nos corpúsculos de Weibel-Palade.

Quando o equilíbrio da célula endotelial é “perturbado", em condições patológicas, ocorre rápida secreção do fvW a partir dos depósitos, o que faz com que os níveis circulantes aumentem consideravelmente. Diversos são os fatores implicados nessa secreção rápida, podendo ser lembrados o aumento do shear stress, a trombina, a fibrina, a serotonina, a vasopressina, endotoxina, catecolaminas, estrógeno, diversas citocinas e componentes do complemento ${ }^{5-10}$. Em vista da possibilidade de estimulação por esses últimos agentes, o fvW é considerado uma "proteína de fase aguda". O retorno da função endotelial às condições de normalidade, é acompanhado de redução dos níveis circulantes aos limites fisiológicos. A atividade antigênica plasmática do fvW (vWF:Ag) é portanto, considerada universalmente como um marcador de disfunção endotelial e assim utilizada em várias doenças ${ }^{11-16}$.

Em pacientes com hipertensão arterial pulmonar, os sintomas aparecem em etapas onde já existe considerável dano à microcirculação naquele território. As lesões predominam em pequenos vasos de resistência e incluem hipertrofia e hiperplasia de células musculares lisas, assim como proliferação fibrocelular intimal, que acaba por ocluir a luz do vaso, com freqüente ocorrência de trombose in situ. Pacientes com hipertensão pulmonar (HP) primária (idiopática) ou secundária a diversas causas, geralmente, são tratados com vasodilatadores, inibidores de agregação plaquetária e anticoagulantes. Não raro, pacientes com formas avançadas da doença tornam-se candidatos a transplante cardiopulmonar, dada a rápida evolução para óbito por hipoxemia e falência ventricular direita.

A sobrevida de pacientes com HP primária é de 68 a $77 \%$ em um ano e diminui para 40 a $56 \%$ em três anos ${ }^{17}$. Diversos índices prognósticos têm sido estabelecidos e usados como marcadores de sobrevida. Em termos de transplante cardiopulmonar, por exemplo, os candidatos ideais seriam aqueles com pouca probabilidade de sobreviver em curto espaço de tempo. Assim, variáveis clínicas, hemodinâmicas, histopatológicas e grau de resposta a vasodilatadores, têm sido utilizados com finalidade prognóstica ${ }^{18}$. As mesmas variáveis podem ser usadas como índices de resposta a novas modalidades terapêuticas introduzidas. No presente estudo, levantamos a hipótese de que o grau de disfunção das células endoteliais, por refletir a gravidade e extensão das lesões microvasculares, poderia ser usado como um índice prognóstico em HP. Assumindo que algumas características do fvW circulante podem ser usadas como marcadores de disfunção endotelial, optamos por testar essa hipótese de maneira não invasiva, através da análise dessa proteína. Assim, o objetivo do presente estudo foi analisar características estruturais e funcionais do fator von Willebrand plasmático em um grupo de pacientes com $\mathrm{HPe}$ verificar possíveis implicações prognósticas relacionadas às variáveis em estudo.

\section{Métodos}

O presente estudo incluiu pacientes com HP, primária ou secundária, admitidos para acompanhamento no INCOR, Hospital das Clínicas da FMUSP. Foram estabelecidos como critérios de inclusão, que os pacientes deveriam ser portadores de HP pré-capilar e apresentar pressão média arterial pulmonar avaliada em repouso, em estudo hemodinâmico, superior a $25 \mathrm{mmHg}$. Foram incluidos pacientes em seguimento clínico por um ano. Indivíduos aguardando transplante permaneceriam no estudo se, nesse período, não fossem submetidos ao procedimento. Os procedimentos de coleta de material foram realizados mediante consentimento após informação e o protocolo do estudo foi apreciado e aprovado pela Comissão Científica e de Ética do INCOR.

Ristocetina e os inibidores de proteases, ácido edético (EDTA), N-etilmaleimida (NEM), fluoreto de fenilmetil sulfonila (PMSF), leupeptina e aprotinina foram adquiridos de Sigma (St. Louis, MO, EUA). Os reagentes para eletroforese e proteína A-sepharose CL4B foram obtidos de Pharmacia Fine Chemicals (Piscataway, NJ, EUA). O kit de imunoeletrodifusão para determinação da atividade antigênica do fvW (Assera-plate vWF) foi de Diagnostica Stago (Asnieres, França). Anticorpo policlonal anti-fvW humano, imunoglobulina $\mathrm{G}$ suína, biotinilada, anti-coelho e complexo avidina-biotina-peroxidase foram adquiridos de Dako Corporation (Carpinteria, CA, EUA). Todos os outros reagentes utilizados foram de alto grau de pureza.

O sangue periférico foi coletado em citrato de sódio 3,8\% na proporção de 9:1 v/v, em presença de inibidores de proteases: EDTA 5mM, NEM 6mM, PMSF 1mM, leupeptina $0,25 \mathrm{mM}$ e aprotinina $20 \mathrm{U} / \mathrm{mL}$. Imediatamente após a coleta, o sangue foi centrifugado por $20 \mathrm{~min}$ a $3.000 \mathrm{x}$ g e o plasma obtido estocado a $-70^{\circ} \mathrm{C}$.

A atividade antigênica do fvW (vWF:Ag) plasmático foi determinada através de imunoeletrodifusão, utilizandose kit apropriado. Os resultados foram expressos como porcentagem de atividade em relação ao pool de plasma normal. Plaquetas normais $\left(5 \times 10^{7}\right.$ células $)$ foram aglutinadas em presença de plasma normal ou plasma de pacientes com HP $(50 \mu \mathrm{L})$ e ristocetina em concentrações de 0,35 a $0,5 \mathrm{mg} / \mathrm{mL}$. A resposta da aglutinação foi determinada pela variação da transmissão de luz, padronizada em 4 min de reação. Os resultados dos pacientes foram comparados com os valores normais no mesmo ensaio e expressos como porcentagem de atividade.

O plasma foi diluído 1:20 em tampão de amostra. Após incubação por $1 \mathrm{~h} \mathrm{a} 56^{\circ} \mathrm{C}$, as amostras foram submetidas a eletroforese em gel de agarose $1 \%$. Foi usada a imunoglobulina M (IgM) como marcador de peso molecular (950 $\mathrm{kDa})$. As proteínas foram transferidas para membrana de nitrocelulose (sistema Multiphor II Novablot LKB, Bromma, Suécia). A membrana foi incubada com anticorpo policlonal anti-fvW (1:150) por $12 \mathrm{~h}$ e em seguida, com anticorpo se- 
cundário (imunoglobulina suína anti-coelho biotinilada) na diluição 1:500 por $2 \mathrm{~h}$. A membrana de nitrocelulose foi então incubada na presença do complexo avidina biotina-peroxidase, na diluição 1:2.500. A proteína foi visibilizada pela adição de diaminobenzidina e $\mathrm{H}_{2} \mathrm{O}_{2}$. A semiquantificação foi feita a 633nm, utilizando-se densitômetro a laser (Ultroscan XL, LKB, Bromma, Suécia). As quatro bandas do fvW com migração mais rápida, com massa molecular pouco acima da IgM usada como padrão, foram consideradas como multímeros de baixo peso molecular. Sua densidade foi expressa como porcentagem em relação ao total de multímeros (MBPM\%).

A purificação do fvW plasmático foi feita por imunoprecipitação. O plasma foi incubado por $1 \mathrm{~h} \mathrm{a} 4^{\circ} \mathrm{C} \mathrm{com} 108 \mu \mathrm{g} /$ $\mathrm{mL}$ (concentração final) de anticorpo anti-fvW humano, em tampão NET-gel(Tris pH 7,5,50mM, NaCl 150mM, Nonidet P-400,1\%), contendo os inibidores de proteases, como descrito anteriormente. A mistura foi então incubada com proteína A-Sepharose por $1 \mathrm{~h}$ a $4^{\circ} \mathrm{C}$. A resina foi lavada, duas vezes, com o mesmo tampão e uma vez com Tris pH 7,5, 10mMe Nonidet P-400,1\%.O fvW foi eluído diretamente em tampão de amostra redutor contendo SDS 2\%. A amostra do fvW purificado foi submetida a eletroforese em gel de poliacrilamida (SDS-PAGE 5,5\%). Após a eletroforese, as proteinas foram transferidas para membrana de nitrocelulose e identificadas por reação de imunoperoxidase. A concentração da subunidade principal e dos fragmentos proteolíticos do $\mathrm{fvW}$ foi determinada por análise densitométrica a laser.

Os resultados obtidos, foram analisados comparativamente entre pacientes e controles no início do estudo e revistos ao final de um ano, comparativamente, entre não sobreviventes e sobreviventes. Após terem sido testadas as variáveis em estudo com relação à sua aderência à distribuição normal, optou-se pela utilização de análise paramétrica na maioria dos procedimentos de quantificação. Os resultados foram expressos em médias e desvios-padrão. Em experimentos onde os valores dos pacientes foram expressos em relação ao controle no mesmo teste, os resultados foram considerados significantes quando os intervalos de confiança de $95 \%$ para as médias não incluíram o valor $100 \%$. As comparações envolvendo controles e grupos diferentes de pacientes, foram feitas com uso de análise de variância. As interdependências entre variáveis, foram testadas com uso de coeficientes de correlação. A influência do tipo de doença sobre a evolução dos pacientes foi testada utilizando-se a distribuição $x^{2}$. As possíveis influências de variáveis contínuas sobre a ocorrência de óbito no período do estudo, foram testadas inicialmente por análise univariada (teste t de Student) e a seguir por análise de regressão logística, com procedimentos "stepwise" para seleção de variáveis (programa SAS/STAT, SAS Institute Inc, Cary, NC, USA). Adotou-se o nível de significância de 0,05 para todos os testes.

\section{Resultados}

Foram incluídos no estudo 40 pacientes, com idades variando entre 1,2 e 45 (mediana 24,5) anos e predominância do sexo feminino (relação entre gêneros de 2,6:1). A pressão média arterial pulmonar variou entre 27 e $112 \mathrm{mmHg}$. Onze pacientes foram identificados como portadores de HP primária e nos demais, a HP secundária estava associada a doença auto-imune (1 caso), síndrome anti-fosfolípides (1), esquistossomose (3) ou cardiopatias congênitas (24). No início do estudo, com apenas uma exceção, todos os pacientes apresentavam sinais e sintomas compatíveis com HP moderada ou acentuada e encontravam-se em classe funcional III ou IV (NYHA).

Pacientes com HP apresentaram valores de vWF:Ag elevados em relação aos controles $(166 \pm 87$ e $87 \pm 23 \%$ de atividade respectivamente, $\mathrm{p}<0,001)$. Os valores foram mais altos nos pacientes com HP primária em relação aos demais ( $231 \pm 89$ e $142 \pm 74 \%$ de atividade, respectivamente, $\mathrm{p}<0,005$ ). Não houve influência do sexo, idade ou pressão arterial pulmonar sobre os resultados observados.

A atividade biológica do fvW, analisada como atividade de cofator da ristocetina, foi avaliada em 27 pacientes. Normalizando-se os resultados para a concentração do fvW presente no plasma, a atividade biológica da proteína mostrou-se reduzida em pacientes com HP (60 a $88 \%$ do valor normal, correspondendo ao intervalo de confiança de 95\% para a média).

A composição multimérica do fvW foi analisada em 27 pacientes. Em relação à proteína presente no plasma normal, observou-se que na maioria desses pacientes, os multímeros de peso molecular mais elevado estavam ausentes na circulação. Em muitos pacientes, também foi observado aumento na concentração de frações de baixo peso molecular (fig. 1-A). Em conseqüência, a concentração relativa de multímeros de baixo peso molecular comparada ao total de

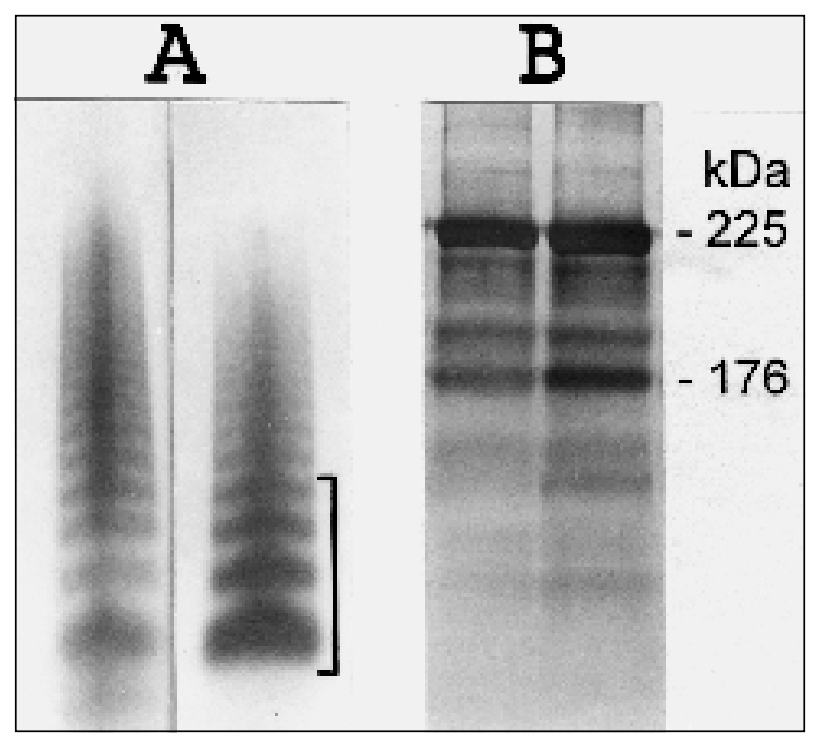

Fig. 1 - A) Estrutura multimérica do fator von Willebrand plasmático: à esquerda, plasma normal; à direita, paciente com HP, apresentando perda de multímeros pesados (parte superior da membrana) e aumento importante na concentração de multímeros de baixo peso molecular (MBPM\%, 4 bandas inferiores em relação ao total, como indicado); B) composição subunitária do fator von Willebrand plasmático: a subunidade principal corresponde à proteína de $225 \mathrm{kDa}$; em relação ao controle (esquerda), o paciente com hipertensão pulmonar apresenta concentrações aumentadas de fragmentos proteolíticos, sobretudo do peptídeo de $176 \mathrm{kDa}$. 
multímeros do fvW (MBPM\%), mostrou-se significativamente aumentada nos pacientes $(56 \pm 12 \%$ vs $35 \pm 12 \%$ em normais, $\mathrm{p}<0,001)$. Não houve diferenças entre pacientes com HP primária e os demais.

A subunidade principal do fvW analisada em 24 pacientes, assim como nos controles, apareceu como uma proteína com migração correspondente a $225 \mathrm{kDa}$. Fragmentos proteolíticos de 189, 176, 150 e $140 \mathrm{kDa}$, estiveram presentes inclusive em plasma normal, mas apareceram com concentrações alteradas em pacientes. Em média, a densidade relativa do fragmento de $176 \mathrm{kDa}$ mostrou-se significativamente aumentada $(\mathrm{p}<0,05)$ (fig. 1-B). As concentrações dos polipeptídeos de 150 e $140 \mathrm{kDa}$ estiveram muito aumentadas em alguns casos. Em conseqüência dessas alterações correspondentes a degradação anormal, a concentração relativa da subunidade principal do fvW tornou-se reduzida nos pacientes $(\mathrm{p}<0,05)$. Nenhuma diferença significante na estrutura da proteína foi verificada, comparando-se pacientes com a forma primária e secundária da doença.

Desejou-se determinar se a atividade biológica do fvW circulante, reduzida em pacientes com HP, poderia estar relacionada ao desaparecimento dos polímeros de alto peso molecular. A atividade biológica correlacionou-se negativamente com magnitude das alterações multiméricas do fator, expressas através do predomínio de polímeros menores em pacientes $(y=1,59-0,02 x, r=-0,54, p<0,005)$. Desejouse ainda verificar a possível influência da degradação proteolítica sobre a atividade biológica do fvW. Foi observada correlação negativa (inversa) entre a atividade biológica da proteína e a concentração de seus fragmentos proteolíticos $\left(\mathrm{y}=165 \mathrm{e}^{0,008 \mathrm{x}}, \mathrm{r}=-0,51, \mathrm{p}<0,015\right)$.

Foram acompanhados pelo período de um ano 40 pacientes, em tratamento exclusivamente clínico. Ao final do período de observação, 11 pacientes faleceram, evento atribuído diretamente à HP, ocorrido em decorrência de hipoxemia e falência circulatória. Os não sobreviventes não diferiram dos demais em relação à idade, sexo e pressão média arterial pulmonar. Entretanto, a evolução foi pior em portadores de HP primária comparados aos demais. Sete entre 11 não sobreviventes, mas apenas quatro entre 29 sobreviventes tinham a forma primária da doença $(\mathrm{p}<0,01)$. Não houve diferenças entre os grupos com relação ao tratamento administrado, baseado no uso de anticoagulantes e antiagregantes plaquetários na maioria dos casos.

Os dados laboratoriais relativos à análise do fvW obtidos no início do período de acompanhamento, foram revistos ao final de um ano. Os pacientes não sobreviventes caracterizaram-se por apresentar valores de vWF:Ag significativamente mais elevados no início do estudo $(257 \pm 85$ vs $132 \pm 59 \%$ de atividade nos sobreviventes, $\mathrm{p}<0,001)$. Todos os seis indivíduos com vWF:Ag > 250\% de atividade evoluíram para óbito. Entre os 27 pacientes nos quais foi possível a análise da estrutura multimérica do fvW, os não sobreviventes também se distinguiram por apresentar alterações mais acentuadas quando vistos inicialmente (MBPM igual a $67 \pm 15$ vs $51 \pm 9 \%$ em sobreviventes, $\mathrm{p}<0,005)$. Todos os quatro pacientes com MBPM $>70 \%$ do total faleceram no período. O seguinte modelo de regressão logística foi ajustado $(\mathrm{p}<0,05)$ para estimar a probabilidade $(\mathrm{P})$ de ocorrência de óbito em um ano, dado um determinado valor de MBPM\%: $\mathrm{P}=\exp (-8,3423+0,1217 \mathrm{MBPM}) / 1+\exp (-8,3423+$ 0,1217MBPM).

O modelo não foi melhorado pela inclusão de outras variáveis. Desta forma, a concentração relativa de multímeros de baixo peso molecular do fvW foi selecionada, como um preditor de ocorrência de óbito em curto prazo, em pacientes com HP pré-capilar.

\section{Discussão}

Nossas observações estão de acordo com comunicações anteriores, no sentido de que em pacientes com HP, as células endoteliais em disfunção sintetizam e secretam grandes quantidades de $\mathrm{fvW}^{19,20}$. Os resultados da atividade de cofator da ristocetina indicam entretanto, que a proteína circulante é funcionalmente anômala. A redução na atividade biológica do fvW observada nesses pacientes parece claramente relacionada ao desaparecimento dos multímeros de alto peso molecular, os mais ativos biologicamente. Em nossos pacientes, observamos que a maior parte da proteína circula sob a forma de multímeros de peso molecular baixo ou intermediário. O aumento na concentração de multímeros de baixo peso molecular, correlacionou-se com a redução na atividade biológica do fator.

O desaparecimento dos grandes polímeros é provavelmente um fenômeno de natureza complexa. Neste estudo, verificamos através da análise de subunidade, que o fvW sofre degradação proteolítica anormal em portadores de HP. O aumento na concentração de fragmentos proteolíticos, correlacionou-se com redução na atividade biológica da proteína. $\mathrm{O}$ fato sugere que a proteólise excessiva pode ser uma das explicações para o desaparecimento dos grandes polímeros, o que por sua vez acarreta perda na atividade biológica. A proteólise do fvW é entendida como um evento extracelular, mediado por enzimas liberadas por leucócitos ${ }^{4}$. Em situações de lesão endotelial, o maior contato de leucócitos com a superfície celular alterada, propicia condições locais para a ocorrência de atividade proteolítica excessiva. Outra possível explicação para a perda de grandes multímeros, seria o envolvimento dos mesmos em microagregados plaquetários, formados em locais de lesão endotelial. Os polímeros maiores seriam desta forma retirados de circulação. Sabe-se que plaquetas estimuladas por trombina absorvem em sua superfície, predominantemente formas de alto peso molecular do fvW ${ }^{21}$. Além disso, em estudo anterior, verificamos que em portadores de HP, microagregados envolvendo plaquetas podem ser identificados em circulação ${ }^{22}$. Finalmente, a inexistência de formas de alto peso molecular no plasma desses pacientes, poderia decorrer de secreção de moléculas incompletamente processadas por células endoteliais lesadas. Neste momento, estamos empenhados na caracterização de oligossacarídeos ligados ao fvW, uma vez que em situações patológicas, defeitos de glicosilação 
poderiam deixar a proteína mais vulnerável à degradação excessiva ${ }^{23}$.

Em pacientes com HP, a ocorrência de disfunção endotelial tem sido fundamentada em diversas observações como alterações em níveis circulantes de trombomodulina e endotelina-1, assim como em aumento de endotelina- 1 e do fvW em artérias pulmonares processadas por imunohistoquímica ${ }^{19,24-26}$. Além disso, já se observaram redução na excreção urinária de 6-ceto-prostaglandina $F_{1 \alpha}$ (metabólito estável da prostaciclina) e perda na capacidade de relaxamento vascular dependente do endotélio ${ }^{27,28}$. Assumindo que as alterações quantitativas e estruturais do fvW possam estar relacionadas à magnitude da disfunção endotelial e provavelmente à gravidade e extensão das lesões vasculares pulmonares, aventamos a hipótese de que tais alterações poderiam ter reflexos sobre a evolução desses pacientes. Os resultados indicam que os pacientes que apresentam alterações mais acentuadas do fvW circulante no início do período de observação, são aqueles com menores possibilidades de sobrevida em um ano. Considerando que os não sobreviventes não diferiram dos sobreviventes quanto ao tipo de tratamento recebido, é possível que a partir de um determinado nível de lesão endotelial e microvascular, expresso por exemplo através de importan- tes alterações quantitativas e qualitativas no fvW plasmático, a doença apresente progressão inexorável, independentemente dos esforços terapêuticos empregados. Gostaríamos de sugerir, com base em nossos achados, que pacientes com vWF:Ag > 250\% de atividade e/ou MBPM > 70\% apresentam alto risco de evolução para óbito em um ano. Por outro lado, é também possível que em alguns dos sobreviventes tenha havido estabilização do processo, com manutenção ou mesmo regressão das alterações bioquímicas. Embora este estudo não tenha sido planejado objetivando a realização de medidas laboratoriais repetitivas, julgamos que esta possibilidade represente a grande vantagem das determinações bioquímicas sobre índices que exigem procedimentos invasivos como o estudo hemodinâmico ${ }^{18}$. Assim, as respostas a determinadas modalidades de tratamento, poderiam ser monitoradas através da utilização de marcadores de lesão endotelial. Por fim, a variável MBPM\% foi selecionada entre outras, como um preditor da ocorrência de óbito em um ano. A aplicação do modelo de regressão logística mostranos que um portador de $\mathrm{HP}$ com fvW circulante apresentando MBPM $>70 \%$ do total, tem mais de $50 \%$ de probabilidade de óbito no $1^{\circ}$ ano de observação. Esta constatação, certamente, deverá ter impacto em decisões terapêuticas, como a possível indicação de transplante cardiopulmonar.

\section{Referências}

1. Ruggeri ZM, Ware J - von Willebrand factor. FASEB J 1993; 7: 308-16.

2. Girma JP, Meyer D, Verweij CL, Pannekoek H, Sixma JJ - Structure-function relationship of human von Willebrand factor. Blood 1987; 70: 605-11.

3. Sadler JE - von Willebrand factor. J Biol Chem 1991; 266: 22777-80.

4. Tsai HM, Nagel RL, Hatcher VB, Sussman II - Endothelial cell-derived high molecular weight von Willebrand factor is converted into the plasma multimer pattern by granulocyte proteases. Biochem Biophys Res Commun 1989; 158: 980-5.

5. Ribes JA, Francis CW, Wagner DD - Fibrin induces release of von Willebrand factor from endothelial cells. J Clin Invest 1987; 79: 117-23.

6. Schores AE, Moldow CF, Rick ME - Interleukin 1 or endotoxin increases the release of von Willebrand factor from human endothelial cells. Br J Haematol 1987; 67: 193-7.

7. Zavoico GB, Ewenstein BM, Schafer AI, Pober JS - IL-1 and related cytokines enhance thrombin-stimulated PGI2 production in cultured endothelial cells without affecting thrombin stimulated von Willebrand factor secretion or platelet-activating factor biosynthesis. J Immunol 1989; 142: 3993-9.

8. Paleolog EM, Crossman DC, McVey JH, Pearson JD- Differential regulation by cytokines of constitutive and stimulated secretion of von Willebrand factor from endothelial cells. Blood 1990; 75: 688-95.

9. Tannenbaum SH, Gralnick HR - Gama-interferon modulates von Willebrand factor release by cultured human endothelial cells. Blood 1990; 75: 2177-84.

10. Van der Poll T, Van Deventer SJH, Pasterkamp G, Van Mourick JA, Búller HR, Ten Cate JW - Tumor necrosis factor induces von Willebrand factor release in healthy humans. Thromb Haemost 1992; 67: 623-6.

11. Kahaleh MB, Osborn I, LeRoy EC - Increased factor VIII/von Willebrand factor antigen and von Willebrand factor activity in scleroderma and in Raynaud's phenomenon. Ann Intern Med 1981; 94: 482-4.

12. Carvalho ACA, Bellman SM, Saullo VJ et al - Altered factor VIII in acute respiratory failure. N Engl J Med 1982; 307: 1113-19.

13. Kelton JG, Moore J, Santos A, Sheridan D - The detection of a platelet-agglutination factor in thrombotic thrombocytopenic purpura. Ann Intern Med 1984; 101: 589-93.

14. Brenner B, Zwang E, Bronshtein M, Seligsohn U - von Willebrand factor multimer patterns in pregnancy-induced hypertension. Thromb Haemost 1989; 62: 715-17.

15. Rubin DB, Wiener-Kronish JP, Murray JF et al - Elevated von Willebrand factor antigen is an early plasma predictor of acute lung injury in nonpulmonary sepsis syndrome. J Clin Invest 1990; 86: 474-80.
16. Blann AD, Midley HS, Burrows Get al - Oxygen free radicals, reperfusion injury, endothelial cells, and von Willebrand factor. Cardiovasc Res 1993; 27: 141-2.

17. American College of Chest Physicians Consensus Statement - Primary pulmonary hypertension. Chest 1993; 104: 236-50.

18. McGoon MD - Prognosis and natural history. In: Rubin LJ, Rich S, eds - Primary Pulmonary Hypertension. New York: Marcel Dekker, 1997: 305-18.

19. Rabinovitch M, Andrew M, Thom Het al - Abnormal endothelial factor VIII associated with pulmonary hypertension and congenital heart defects. Circulation 1987; 76: 1043-52.

20. Lopes AAB, Maeda NY, Aiello VD et al - Abnormal multimeric and oligomeric composition is associated with enhanced endothelial expression of von Willebrand factor in pulmonary hypertension. Chest 1993; 104: 1455-60.

21. Fernandez Ginsberg MH, RuggeriZM, BatleFJ,Zimmerman TS -

Multimeric structure of platelet factor VIII/von Willebrand: The presence of larger multimers and their association with thrombin-stimulated platelets. Blood 1982; 60: 1132-8.

22. Lopes AAB, Maeda NY, Almeida A, Jaeger R, Ebaid M, Chamone DF-Circulating platelet aggregates indicative of in vivo platelet activation in pulmonary hypertension. Angiology 1993; 44:701-6.

23. Berkowitz SD, Federici AB - Sialic acid prevents loss of large von Willebrand factor multimers by protecting against amino-terminal protelytic cleavage. Blood 1988; 72: 1790-6.

24. Cacoub P, Karmochkine M, Dorent R et al - Plasma levels of thrombomodulin in pulmonary hypertension. Am J Med 1996; 101: 160-4.

25. Yoshibayashi M, Nishioka K, Nakao K et al - Plasma endothelin concentrations in patients with pulmonary hypertension associated with congenital heart defects. Evidence for increased production of endothelin in pulmonary circulation. Circulation 1991; 84: 2280-5

26. Giaid A, Yanagisawa M, Langleben D et al - Expression of endothelin-1 in the lungs of patients with pulmonary hypertension. N Engl J Med 1993; 328: 17329.

27. Christman BW, Mc Pherson CD, Newman JH et al - An imbalance between the excretion of thromboxane and prostacyclin metabolites in pulmonary hypertension. N Engl J Med 1992; 327: 70-5.

28. Uren NG, Ludman PF, Crake T et al-Response of the pulmonary circulation acetylcholine, calcitonin gene-related peptide, substance $P$ and oral nicardipine in patients with primary pulmonary hypertension. J Am Coll Cardiol 1992; 19: 83541 . 